\title{
Conciencia ambiental en estudiantes universitarios de una universidad pública de Lima
}

Environmental awareness in university students of a public university in Lima

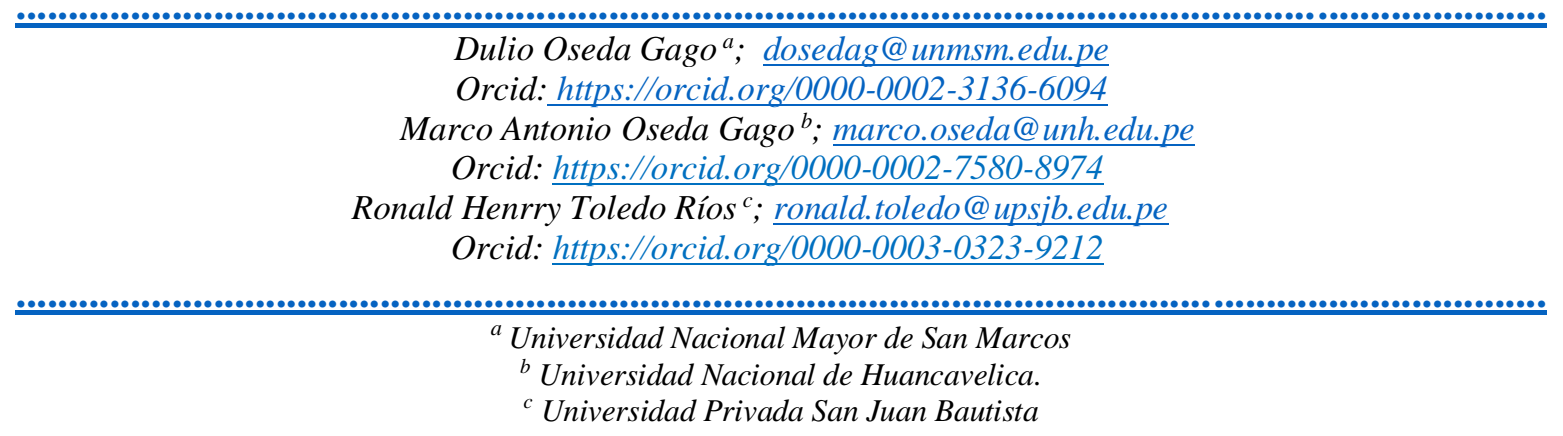

Recibido Julio/23/2020 • Aceptado: Agosto/02/2020 •Publicado: Septiembre/30/2020

\section{RESUMEN}

El estudio realizado corresponde a una investigación de carácter cuantitativo descriptivo en una muestra probabilística de 304 estudiantes de la Facultad de Educación de la Universidad Nacional Mayor de San Marcos. El objetivo de la investigación consistió en describir los niveles predominantes de conciencia ambiental de los estudiantes de la Facultad de Educación de la Universidad Nacional Mayor de San Marcos de Lima; y la investigación por ser descriptivo metodológicamente no tiene hipótesis. La investigación fue del tipo básica, nivel descriptivo, de diseño: muestra observación (M-O). La muestra probabilística estuvo constituida por 304 estudiantes de la Facultad de Educación de la mencionada universidad; se utilizó la técnica de la encuesta con su instrumento el cuestionario de encueta el cual se confiabilizó y validó antes de su aplicación. El aporte más importante de la presente investigación considera que en base a los datos analizados y procesados, con un nivel de significancia del $5 \%$ el nivel predominante de conciencia ambiental de los estudiantes de la Facultad de Educación de la Universidad Nacional Mayor de San Marcos fue alto.

Palabras claves: Actitud ambiental, conciencia ambiental, cognitivas, afectivas, conductuales y estudiantes.

${ }^{\mathrm{a}}$ Dulio Oseda - Gago; ${ }^{\mathrm{b}} \mathrm{M}$ Marco Antonio Oseda - Gago y ${ }^{\mathrm{c}}$ Ronald Henrry Toledo - Ríos

Articulo Protegido por Licencia Creative Commons: BY-NC-ND / Protected by Creative Commons: BY-NC-ND.

Sendas es una revista de acceso abierto / Sendas is an Open Access Journal. 


\section{ABSTRACT}

The study carried out corresponds to a quantitative-descriptive investigation in a probabilistic sample of 304 students from the Faculty of Education of the Universidad Nacional Mayor de San Marcos. The objective of the research was to describe the predominant levels of environmental awareness of the students of the Faculty of Education of the National University of San Marcos de Lima; and because the research is methodologically descriptive, it has no hypothesis. The research was of the basic type, descriptive level, of design: observation sample (M-O). The probabilistic sample consisted of 304 students from the Faculty of Education of the mentioned university; The survey technique was used with its instrument the survey questionnaire, which was trusted and validated before its application. The most important contribution of this research considers that based on the data analyzed and processed, with a significance level of $5 \%$, the predominant levels of environmental awareness of the students of the Faculty of Education of the National University of San Marcos was High.

Keywords: environmental attitude, environmental, cognitive, emotional, behavioral and students consciousness.

\section{Introducción}

A nivel internacional se puede apreciar que nuestro planeta está enfermo; los seres humanos lo hemos maltratado, sometiéndolo a una grave sobre explotación, contaminándolo de muchas formas. La producción de gases que polucionan el aire; los residuos sólidos y líquidos que son arrojados al agua y al suelo sin ningún control; el exceso de ruido y de imágenes publicitarias contribuyen con el incremento de la contaminación ambiental y el aumento de varias de las enfermedades que nos aquejan.

Además, se estima que cada habitante de América Latina y El Caribe produce entre medio kilogramo y un kilogramo de basura por día. O sea que una familia de cinco personas genera en un mes de 100 a 160 kilogramos de desperdicios", según Cristian Frers En Chile,

${ }^{\mathrm{a}}$ Dulio Oseda - Gago; ${ }^{\mathrm{b} J}$ Marco Antonio Oseda - Gago y ${ }^{\mathrm{c}}$ Ronald Henrry Toledo - Ríos

Articulo Protegido por Licencia Creative Commons: BY-NC-ND / Protected by Creative Commons: BY-NC-ND.

Sendas es una revista de acceso abierto / Sendas is an Open Access Journal. 
el creciente desarrollo de su economía, ha traído consigo un considerable aumento en la generación de residuos.

En Colombia, una persona, en sus labores cotidianas, produce $0.6 \mathrm{Kg} / \mathrm{hab}$ en promedio de residuos sólidos por día, se encuentra muy por debajo del promedio internacional, que es de $1 \mathrm{~kg}$ diario; y la economía colombiana es incipiente en cuanto a procesos tecnificados se refiere. Una de las preocupaciones más importante de nuestro tiempo es la calidad ambiental del entorno. Como es bien conocido en los últimos 150 años, el planeta ha cambiado su estructura natural de la atmósfera e hidrosfera más que en todo el tiempo que tiene en existir.

\section{La Directora Ejecutiva de la} Fundación Ambiente y Recursos Naturales (FARN), María Eugenia Di Paola explica que la basura, su tratamiento y disposición, será un problema a resolver en la década que viene. Di Paola quien es experta en derecho de los Recursos Naturales e hizo un máster en Derecho Ambiental expresa que, en primer término, hace falta revertir el modelo de contaminación imperante por uno diferente, que dé prioridad a la restauración y prevención. "Esto implica trabajar en la gestión integral de los residuos que incluyen el reciclado, revalorización y reutilización de los elementos que consumimos".

La basura doméstica es un problema para cualquier administración pública, pues, entre los daños que desarrolla la sociedad industrializada, uno de los mayores es la proliferación de este tipo de residuos en las ciudades. El volumen del desecho que debe ser manejado, es importante, que la extensión de los tiraderos no puede considerarse ya como una solución aceptable.

Este problema representa un peligro real para la salud de las poblaciones ya que provoca olores, plagas y enfermedades. Según el análisis estadístico del comportamiento de los residuos sólidos domiciliarios en una comunidad urbana que investigó Sara Ojeda Benítez y otros.

El reciclaje de residuos sólidos se considera una estrategia importante para contribuir al fortalecimiento de la cultura ambientalista, en el aprovechamiento sustentable de los escasos recursos naturales del mundo y evitar los conflictos entre las naciones.

Algunos países desarrollados son vanguardistas en el reciclaje, pero en contraste, proponen transferir tecnología

${ }^{\mathrm{a}}$ Dulio Oseda - Gago; ${ }^{\mathrm{b}} \mathrm{M}$ Marco Antonio Oseda - Gago y ${ }^{\mathrm{c}}$ Ronald Henrry Toledo - Ríos Articulo Protegido por Licencia Creative Commons: BY-NC-ND / Protected by Creative Commons: BY-NC-ND. Sendas es una revista de acceso abierto / Sendas is an Open Access Journal. 
obsoleta a los países en vías de desarrollo. El reciclaje de residuos sólidos también es un asunto socio-político, que obliga a países en desarrollo a establecer leyes para su gestión y de esa forma proteger un ambiente de calidad.

Para la formación de una conciencia ambiental es necesario el desarrollo de la ética ambiental y la educación ambiental, como procesos indispensables en la conformación de dicha conciencia ambiental, la combinación de ambos procesos posibilita un óptimo desenvolvimiento de este objetivo, mediante los cuales se introducen un conjunto de principios y valores morales en las conductas humanas que establecen la autodeterminación y autoconfianza del individuo para conservar y mejorar la calidad ambiental y los recursos naturales, con el fin de que el hombre no se sienta independiente de la naturaleza sino como parte integradora de la misma, como el único agente posible de reparar el gran daño ocasionado y posibilitar una alta calidad de vida en nuestra sociedad.

A nivel nacional, se puede apreciar que en el Perú hay serios problemas ambientales que justifican la creación de un Ministerio del Ambiente, por ejemplo: el incremento en los índices de contaminación del agua, tierra y aire; el crecimiento desordenado de las ciudades; la eliminación de los residuos líquidos y sólidos; el hacinamiento y las viviendas con materiales inadecuados; la presencia de gérmenes patógenos debido a la carencia de una infraestructura básica y de servicios de agua potable, desagüe y servicios de recolección de basura.

Según el ex ministro del Ambiente, Antonio Brack, la mayor contaminación no es generada por las empresas industriales y mineras, sino por los ciudadanos de a pie: "Tenemos que ser conscientes de que no es la fábrica ni la minería los que más contaminan en el Perú. Somos nosotros, los ciudadanos, quienes contaminamos más".

Lo concreto es que la situación ambiental en el Perú es más que grave. Brack reveló que el $83 \%$ de los desperdicios que se generan en el Perú es lanzado al medio ambiente.

Solo en lo que respecta a residuos sólidos, en el ámbito municipal urbano se generan 12,986 toneladas de basura al día, de las cuales la cuarta parte podría ser reciclada, de acuerdo con información del Ministerio de Ambiente (MINAM).

a Dulio Oseda - Gago; ${ }^{\mathrm{b} J}$ Marco Antonio Oseda - Gago y ${ }^{\mathrm{c}}$ Ronald Henrry Toledo - Ríos

Articulo Protegido por Licencia Creative Commons: BY-NC-ND / Protected by Creative Commons: BY-NC-ND.

Sendas es una revista de acceso abierto / Sendas is an Open Access Journal. 
En el último informe que el Perú envió a la Convención Marco de las Naciones Unidas sobre Cambio Climático (UNFCCC), el Minam reconoció que el $70 \%$ de la basura recolectada a nivel nacional es llevado a los botaderos no autorizados o es quemado directamente, lo cual propicia la emisión de dióxido de carbono $\left(\mathrm{CO}_{2}\right)$ y de gas metano (21 veces más contaminante que el $\mathrm{CO}_{2}$ ).

Para revertir este problema, Brack incidió en la necesidad de forjar una educación ambiental. "Desde la escuela tenemos que enseñar a los niños a cuidar el ambiente para que tengan en la mente que la patria no es un basurero. Así lograremos un Perú limpio y sostenible", añadió.

La problemática del manejo de residuos sólidos en nuestro país tiene diferentes orígenes, dentro de los que se destacan: la poca conciencia ambiental de productores y consumidores, la baja capacidad de inversión del país en general, el crecimiento de una cultura orientada al consumo, una falta de educación formal sobre el tema y un marco normativo insuficiente y desactualizado.

La realidad problemática que se presenta a nivel nacional es por la falta de contenedores de residuos sólidos en distintos ciudades y distritos. También es un problema la falta de camiones recolectores; y poca educación de cultura ambiental de sus pobladores.

Reciclar los residuos sólidos es, sin duda, un paso importante para contribuir con el medio ambiente. Pero no basta con apoyar a los recicladores o lograr que el Estado se involucre en esta tarea. Es necesario conseguir que el ciudadano de a pie sea consciente de su propio papel en este circuito, de cuánto puede hacer con sólo segregar los residuos en el hogar o ejercer un consumo consciente de los recursos naturales. Esto se llama sensibilización ambiental.

La sensibilización ambiental implica un cambio cultural que sólo podrá conseguirse a través del tiempo. Aquí la educación ambiental resulta clave, y supone una serie de variables como la formación de los propios educadores, la adecuación de los contenidos a los distintos espacios geográficos del país y el estímulo a la participación directa, al debate y la generación de alternativas según cada contexto social.

Al hablar de conciencia ambiental pensamos en los circuitos de la educación formal, en primer lugar, pero esta tarea no

${ }^{\mathrm{a}}$ Dulio Oseda - Gago; ${ }^{\mathrm{b} J}$ Marco Antonio Oseda - Gago y ${ }^{\mathrm{c}}$ Ronald Henrry Toledo - Ríos Articulo Protegido por Licencia Creative Commons: BY-NC-ND / Protected by Creative Commons: BY-NC-ND. Sendas es una revista de acceso abierto / Sendas is an Open Access Journal. 
puede agotarse en esa instancia, sino que tiene que extenderse al conjunto de la sociedad. Para eso, un vehículo primordial es el propio Estado que debe, en primer lugar, delinear la estrategia del sector Educación en esta tarea y coordinar el desarrollo de programas en otros sectores de su competencia, como salud, agricultura, pesca, etc., destinando los recursos para hacerlo posible. En esto es necesario incluir también a las entidades públicas y municipios, en particular aquellas empresas municipales que tienen que ver directamente con la gestión de residuos sólidos.

A nivel institucional, el problema real que presenta la Facultad de Educación, con respecto al tratamiento de residuos sólidos es muy alarmante, debido al poco conocimiento de las causas y consecuencias de la contaminación ambiental.

Además, la falta de conciencia ambiental que presentan los integrantes de la comunidad educativa; limita la recolección y tratamiento adecuado de los residuos sólido, produciendo contaminación ambiental, situación que repercute en la salud de los estudiantes y el calentamiento global del ambiente; problema que se agrava por la ausencia de conectores que sirve para recolectar los residuos sólidos, provocando en los estudiantes el arrojo de las basuras en los patios, pasadizos y salones; el mayor problema es la indiferencia que muestran los docentes en la solución de este problema.

$$
\text { Los educadores somos los }
$$

responsables directos de velar por una escuela limpia y saludable, tenemos la obligación moral de concientizar a nuestros alumnos, para que ellos asuman su rol, la de preservar y conservar el medio ambiente.

Problema: ¿Cuáles son los niveles predominantes de conciencia ambiental de los estudiantes de la Facultad de Educación de la Universidad Nacional Mayor de San Marcos de Lima?

Objetivo: Describir los niveles predominantes de conciencia ambiental de los estudiantes de la Facultad de Educación de la Universidad Nacional Mayor de San Marcos de Lima.

Respecto al marco teórico, los antecedentes internacionales, el trabajo de Ardaiz, (2016), realizó la investigación: "Indicadores de desarrollo sostenible: la situación de Navarra", en el Instituto de Estadística de Navarra, Gobierno de Navarra, González, (2016), sustentó la tesis doctoral

a Dulio Oseda - Gago; ${ }^{\mathrm{b} J}$ Marco Antonio Oseda - Gago y ${ }^{\mathrm{c}}$ Ronald Henrry Toledo - Ríos Articulo Protegido por Licencia Creative Commons: BY-NC-ND / Protected by Creative Commons: BY-NC-ND. Sendas es una revista de acceso abierto / Sendas is an Open Access Journal. 
titulada "Medición del desarrollo sostenible a través de índices sintéticos: diseño y aplicación a la Unión Europea" al Departamento de Economía Aplicada de la Universidad de Coruña en España, Castro, (2012), en su tesis doctoral titulada "Indicadores de desarrollo sostenible urbano, la aplicación para Andalucía" desarrollado en la Universidad de Málaga en España.

Y como antecedente nacional, se tuvo a López, (2017) quien realizó la investigación: "Evaluación Integral para el desarrollo sostenido en la ciudad de Huancayo", desarrollado en la Escuela de Post Grado de la Universidad Nacional del Centro del Perú, luego también se tiene a De Cruz (2016) en Venezuela en su investigación: "Reciclaje en la escuela básica Nacional" y finalmente Rosales (2017) quien realizó su trabajo de investigación sobre una: "Propuesta de un programa de actividades que facilite la construcción de una conciencia ecológica en estudiantes universitarios de la selva central", esto también en la Escuela de Posgrado de la Universidad Nacional del Centro del Perú.

Respecto a las bases teóricas científicas, atendiendo a las exigencias del desarrollo teórico, se abordó diferentes perspectivas expuestas por varios autores; así como, se mencionan diferentes definiciones que permiten promover el desarrollo de la conciencia ambiental en los estudiantes de primaria.

\section{La sensibilización, según Beltrán y} Pérez (2010), "El principio de sensibilización implica que el profesor debe lograr un contexto mental adecuado dentro del alumno, de manera que éste tenga conciencia clara de lo que ha de conseguir (estado de meta), conozca su estado inicial de conocimientos (estado de partida) y se sienta sensibilizado para transformar el estado de partida en estado de meta. Para ello se necesita motivación, actitudes positivas y control emocional".

Asimismo, reciclar es usar los materiales una y otra vez para hacer nuevos productos, ofrece mínimos problemas de contaminación y mayor facilidad para su recuperación. Por ejemplo: El reciclado de papel, evita la tala indiscriminada de árboles.

Reciclar es un proceso simple que nos puede ayudar a resolver muchos de los problemas creados por la forma de vida moderna. Se pueden salvar grandes cantidades de recursos naturales no renovables cuando en los procesos de producción se utilizan materiales reciclados. Los recursos

a Dulio Oseda - Gago; ${ }^{\mathrm{b} J}$ Marco Antonio Oseda - Gago y ${ }^{\mathrm{c}}$ Ronald Henrry Toledo - Ríos

Articulo Protegido por Licencia Creative Commons: BY-NC-ND / Protected by Creative Commons: BY-NC-ND.

Sendas es una revista de acceso abierto / Sendas is an Open Access Journal. 
renovables, como los árboles, también pueden ser salvados.

La utilización de productos reciclados disminuye el consumo de energía. Cuando se consuman menos combustibles fósiles, se generará menos $\mathrm{CO}_{2}$ y por lo tanto habrá menos lluvia ácida y se reducirá el efecto invernadero. En el aspecto financiero, podemos decir que el reciclaje puede generar muchos empleos.

Los residuos sólidos según Betacourt, (2004, p. 8) señala que: “Los desechos sólidos son un conjunto de materiales sólidos de origen orgánico e inorgánico que no tienen utilidad práctica para la actividad que las producen, siendo procedentes de las actividades domésticas, comerciales y de todo tipo que se producen en una comunidad con excepción de las excretas humanas".

La Ley $N^{\circ}$ 27314, Ley General de Residuos Sólidos, en el artículo $14^{\circ}$ define que los residuos sólidos son aquellas sustancias, productos o subproductos en estado sólido o semisólido de los que su generador dispone, o está obligado a disponer.

Aguilar y Salas (2017, pp. 9 - 59), al realizar la síntesis de su trabajo, podemos rescatar la siguiente: Pensar en la basura nos genera un rechazo inmediato hacia ésta; sin embargo, aunque no se quiera, tenemos que vivir con ella, y no sólo en nuestros hogares, sino a la vuelta de cualquier esquina, en las orillas de las carreteras o de los muchos tiraderos que proliferan por todas partes.

Aye y Widjaya (2016) clasifican los desechos sólidos en dos grandes grupos, orgánicos e inorgánicos. Los orgánicos que incluyen los putrescibles (que se degradan rápidamente y producen mal olor durante la descomposición), papel, cartón, caucho y madera. Los inorgánicos comprenden plásticos, vidrio, metal y otros, se clasifica de la siguiente manera:

Por su Composición:

- Desechos Orgánicos: Es de origen biológico, fue parte de un ser vivo (Hojas, cáscaras y desechos de la fabricación de alimentos en el hogar, etc.)

- Desechos inorgánicos: Es de origen industrial, o resultado de un proceso no natural (plástico, telas sintéticas, etc.).

- Desechos peligrosos: Puede ser de origen biológico o no, que constituye un peligro potencial y debe ser

a Dulio Oseda - Gago; ${ }^{\mathrm{b} J}$ Marco Antonio Oseda - Gago y ${ }^{\mathrm{c}}$ Ronald Henrry Toledo - Ríos Articulo Protegido por Licencia Creative Commons: BY-NC-ND / Protected by Creative Commons: BY-NC-ND. Sendas es una revista de acceso abierto / Sendas is an Open Access Journal. 
tratado de manera especial; por ejemplo: el material médico es infeccioso, el material radioactivo, ácidos y sustancias químicas, entre otros son corrosivos.

- De clasificación dudosa: El papel y el cartón son de origen orgánico, sin embargo, para propósitos de reciclaje deben ser tratados como inorgánicos por el proceso particular que se les da. Pero los papeles y servilletas con desechos de comida se consideran como material orgánico.

- Otros tipos de desechos, como los propios del metabolismo humano, también son orgánicos, sin embargo, son manejables a través de las redes de saneamiento y no a través de recolección y disposición final.

Por su Origen:

- Desechos domésticos: Es generado en los hogares, por las actividades propias de las personas en sus viviendas.

- Desechos urbanos: Es el correspondiente a las poblaciones, como desechos de parques y jardines, inmobiliario urbano inservible, etc.

\section{https://doi.org/10.47192/rcs.v1i3.46}

- Desechos industriales: Es la generada por las industrias como resultado de sus particulares procesos de producción.

Según la Ley $\mathrm{N}^{\mathrm{o}}$ 27314, de los Residuos Sólidos y su Reglamento, los clasifica tomando en cuenta dónde se producen o generan los residuos.

Esta clasificación se llama, "Tipos de residuos sólidos según el origen de su generación" y es muy importante porque nos permite saber cómo separarlos para su reciclaje.

¿Qué es conciencia ambiental? Es un proceso dinámico y participativo, que busca despertar en la población un conocimiento que le permita identificarse con la problemática Ambiental tanto a nivel general (mundial), como a nivel especifico (medio donde vive).

Para Morachimo (2009), la conciencia ambiental es entendida como "el nivel ético moral que te permite optar libre y críticamente ante acciones de conservación, protección y uso sostenible del ambiente, el cual ha sido fomentado a través de actividades que te motiven, te permitan adquirir conocimiento, te facilite la experimentación, evoque tu

${ }^{\mathrm{a}}$ Dulio Oseda - Gago; ${ }^{\mathrm{b} J}$ Marco Antonio Oseda - Gago y ${ }^{\mathrm{c}}$ Ronald Henrry Toledo - Ríos

Articulo Protegido por Licencia Creative Commons: BY-NC-ND / Protected by Creative Commons: BY-NC-ND.

Sendas es una revista de acceso abierto / Sendas is an Open Access Journal. 
compromiso y te permita la acción sobre tu entorno local".

Sin embargo, para Corraliza, et al (2004) el concepto de conciencia ambiental es utilizado genéricamente para describir el conjunto de creencias, actitudes, normas y valores relacionados con el medio ambiente y es una referencia prácticamente constante en múltiples estudios sobre educación ambiental.

Para CONAM (2017), la conciencia ambiental es la formación de conocimientos, interiorización de valores y la participación en la prevención y solución de problemas ambientales.

A través de la Educación Ambiental se promueven el desarrollo de actitudes para la toma de Conciencia sobre la necesidad de buscar un desarrollo sostenible, mediante la adquisición de valores y hábitos de participación en la protección del medio ambiente.

Toda educación ambiental debe promover una Conciencia Ambiental que garantice el cambio cultural y el desarrollo humano integral (Bermúdez, 2003).

En forma general, Conciencia Ambiental significa conocer la naturaleza y nuestro entorno para respetarlo y cuidarlo, a fin de que la generación venidera pueda disfrutarlo.

Entre las dimensiones tenemos: cognitiva, afectiva y conductual, que se encuentran integradas:

- Cognitiva: El desarrollo de lo cognitivo debe ser el centro del proceso de enseñanza aprendizaje del trabajo docente. Las capacidades cognitivas se desarrollan a través del proceso de información, análisis, argumentación, para comprender, y se manifestar en la acción.

- Afectiva: Toda actitud incluye componentes como sentimientos y afectos que acompañan a la idea $\mathrm{y}$ creencia. En ese sentido, se considera que el componente afectivo de una actitud está ligado directamente a la tendencia hacia la acción.

- Conductual: También considera que los estudios realizados que se centran en creencias e ideas, son factores que pueden implantar una percepción del mundo desde una óptica pro ambiental.

En el gráfico se aprecia la auto dependencia entre las dimensiones y sus respectivos

a Dulio Oseda - Gago; ${ }^{\mathrm{b} J}$ Marco Antonio Oseda - Gago y ${ }^{\mathrm{c}}$ Ronald Henrry Toledo - Ríos Articulo Protegido por Licencia Creative Commons: BY-NC-ND / Protected by Creative Commons: BY-NC-ND. Sendas es una revista de acceso abierto / Sendas is an Open Access Journal. 
Sendas. 1(3), Julio - Septiembre, 2020.

URL: https://www.revistas.infoc.edu.pe/index.php/sendas

Email: revistasendas@infoc.edu.pe

indicadores, los cuales se representan como componentes para la creación de conciencia ambiental.

\section{Figura 1.}

Auto dependencia entre dimensiones e indicadores de Conciencia Ambiental

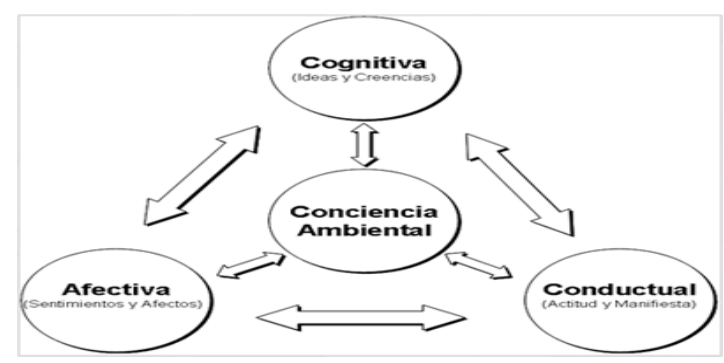

Fuente: Rodríguez (2007).

Rodríguez, (2007) demostró experimentalmente que el componente cognitivo y el afectivo tienden a ser coherentes entre sí; este investigador, a través de los resultados de sus estudios, evidenció igualmente que los componentes conductuales, como las actitudes que tienen baja consistencia afectiva - cognitiva son relativamente inestables en el tiempo.

En síntesis, la variable 1 conciencia ambiental es entendida como "el nivel ético moral que te permite optar libre y críticamente ante acciones de conservación, protección
Vol. 1, N³, pp. 1-18 Septiembre 2020 ISSN $2708-6380$ https://doi.org/10.47192/rcs.v1i3.46

y uso sostenible del ambiente, el cual ha sido fomentado a través de actividades que te motiven, te permitan adquirir conocimiento, te facilite la experimentación, evoque tu compromiso y te permita la acción sobre tu entorno local. Morachimo (2009).

\section{Tabla 1}

Estadios del desarrollo cognitivo según Piaget, desarrollo moral de Kohlberg en la construcción de conciencia ambiental

\begin{tabular}{|c|c|l|}
\hline $\begin{array}{c}\text { Estadios del } \\
\text { desarrollo } \\
\begin{array}{c}\text { cognitivo según } \\
\text { Piaget }\end{array}\end{array}$ & $\begin{array}{c}\text { Fases del } \\
\text { desarrollo moral } \\
\text { según Kohlberg }\end{array}$ & $\begin{array}{l}\text { Etapas en la } \\
\text { construcción de } \\
\text { conciencia } \\
\text { ambiental }\end{array}$ \\
\hline $\begin{array}{c}\text { Sensorio - } \\
\text { motor } \\
\text { preoperacional }\end{array}$ & $\begin{array}{c}\text { Moral } \\
\text { Heterónoma }\end{array}$ & $\begin{array}{l}\text { Sensibilización - } \\
\text { motivación }\end{array}$ \\
\hline \multirow{2}{*}{$\begin{array}{c}\text { Operaciones } \\
\text { concretas }\end{array}$} & $\begin{array}{c}\text { Moral } \\
\text { Instrumental }\end{array}$ & $\begin{array}{l}\text { Conocimiento - } \\
\text { Información }\end{array}$ \\
\cline { 2 - 3 } & $\begin{array}{c}\text { Moral } \\
\text { Conformista }\end{array}$ & $\begin{array}{l}\text { Experimentación - } \\
\text { interacción }\end{array}$ \\
\hline \multirow{2}{*}{$\begin{array}{c}\text { Operaciones } \\
\text { formales }\end{array}$} & $\begin{array}{c}\text { Moral del Deber } \\
\text { Contractual }\end{array}$ & $\begin{array}{l}\text { Capacidades } \\
\text { Compromión - }\end{array}$ \\
\cline { 2 - 3 } & Moral Universal & $\begin{array}{l}\text { Acción voluntaria - } \\
\text { Participación }\end{array}$ \\
\cline { 2 - 3 } & & \\
\hline
\end{tabular}

Fuente. (Morachimo 2009).

\section{$\underline{\text { Metodología }}$}

La investigación según (Kerlinger y Lee, 2002) fue del tipo de estudio básica. El nivel de investigación fue el descriptivo. El

a Dulio Oseda - Gago; ${ }^{\mathrm{b} J}$ Marco Antonio Oseda - Gago y ${ }^{\mathrm{c}}$ Ronald Henrry Toledo - Ríos

Articulo Protegido por Licencia Creative Commons: BY-NC-ND / Protected by Creative Commons: BY-NC-ND.

Sendas es una revista de acceso abierto / Sendas is an Open Access Journal. 
diseño de investigación utilizado fue el Descriptivo - Simple: Muestra observación.

$$
\mathrm{M}-\mathrm{O}
$$

Donde:

\section{M: Muestra}

\section{O: Observación.}

Población: En el caso de nuestra investigación, la población estuvo conformado por los 540 estudiantes de la Facultad de Educación de la Universidad Nacional Mayor de San Marcos.

Muestra: Fue probabilístico y estuvo conformada por 304 estudiantes.

Para la materialización de la investigación se hizo uso del método científico. A decir de Ander (1984) sostiene que el método científico comprende un conjunto de normas que regulan el proceso de cualquier investigación que merezca ser calificada como científica. Y como método específico, al método descriptivo. Según Oseda (2008) el método descriptivo se preocupa primordialmente describir algunas características fundamentales de conjuntos homogéneos de fenómenos, utilizando criterios sistemáticos para destacar los elementos esenciales de su naturaleza.
Caracteriza un fenómeno o una situación concreta indicando sus rasgos diferenciadores.

Respecto a las técnicas e instrumentos de recolección de datos, se hizo uso de la técnica de la encuesta, según Oseda (2008) la técnica de la encuesta está destinada a obtener datos de varias personas cuyas opiniones impersonales interesan al investigador. Para ello a diferencia de la entrevista se utilizan un listado de preguntas escritas que se entregan a los sujetos a fin de que las contesten igualmente por escrito.

Como instrumentos de investigación se utilizó el cuestionario de encuesta para recoger la información sobre la conciencia ambiental en los estudiantes de la mencionada facultad.

Método de análisis de datos, según (Hernández, Fernández, y Bautista, 2016) se utilizó los siguientes estadígrafos:

- Las tablas de distribución de frecuencias (absoluta y la porcentual) con las que se procesaron los ítems del cuestionario de encuesta.

\footnotetext{
Asimismo se tuvo en cuenta los gráficos estadísticos, entre ellos el histograma de frecuencias que sirvió para visualizar e interpretar los resultados.
}

Oseda - Gago: b J Marco Antonio Oseda - Gago yc Ronald Henrry Toledo Articulo Protegido por Licencia Creative Commons: BY-NC-ND / Protected by Creative Commons: BY-NC-ND. Sendas es una revista de acceso abierto / Sendas is an Open Access Journal. 


\section{$\underline{\text { Resultados }}$}

La variable conciencia ambiental está conformado por tiene 3 dimensiones, la misma que fue aplicada a los 304 estudiantes. Veamos en primer lugar a la variable Conciencia Ambiental de manera general:

\begin{tabular}{|l|c|c|}
\hline \multicolumn{3}{|c|}{ Niveles de Conciencia Ambiental } \\
\hline \multicolumn{1}{|c|}{ Niveles } & Frecuencia & Porcentaje \\
\hline Muy deficiente & 8 & 2.63 \\
\hline Deficiente & 24 & 7.89 \\
\hline Regular & 80 & 26.32 \\
\hline Buena & 180 & 59.21 \\
\hline Muy buena & 12 & 3.95 \\
\hline Total & 304 & 100.00 \\
\hline
\end{tabular}

Fuente: Base de datos de los investigadores.

De la tabla 2, se puede apreciar que, después de haber evaluado con el cuestionario de encuesta a los 304 estudiantes de la muestra de estudio, se tiene que la gran mayoría de ellos que son 180 estudiantes y que representa el $59,21 \%$ posee un nivel bueno y/o alto de conciencia ambiental, luego tenemos a 80 estudiantes que representa $26,32 \%$ en el nivel regular, luego a 24 estudiantes que representa el 7,89\% en el nivel deficiente, asimismo a 12 estudiantes que representa el 3,95\% en el nivel muy bueno y finalmente solo a 8 estudiante que representa el 2,63 en el nivel muy deficiente. Cabe mencionar que la gran mayoría de los estudiantes evaluados estuvieron en el nivel bueno o alto, el cual es un buen indicador.

Ahora se aprecia las dimensiones de la conciencia ambiental en la muestra de estudio:

Tabla 3

\begin{tabular}{|l|c|}
\hline \multicolumn{2}{|c|}{$\begin{array}{c}\text { Resumen del desarrollo de la conciencia } \\
\text { ambiental }\end{array}$} \\
\hline \multicolumn{1}{|c|}{ Habilidades Sociales } & Porcentaje \\
\hline Componente cognitivo & $40.04 \%$ \\
\hline Componente afectivo & $38.40 \%$ \\
\hline Componente conductual & $22.56 \%$ \\
\hline \multicolumn{1}{|c|}{ Total } & $100.00 \%$ \\
\hline
\end{tabular}

Fuente: Base de datos de los investigadores.

De la tabla 3, se puede apreciar, que, de los 304 estudiantes de la muestra de estudio, el componente más desarrollado fue el componente cognitivo con el 40,04\%, luego el componente afectivo con el 38,40\% y finalmente el componente conductual con el $22,56 \%$. Lo cierto es que los estudiantes conocen muy bien el cuidado del medio ambiente, pero generalmente no lo practican; o sea no desarrollan a plenitud el componente conductual.

a Dulio Oseda - Gago; ${ }^{\mathrm{b} J}$ Marco Antonio Oseda - Gago y ${ }^{\mathrm{c}}$ Ronald Henrry Toledo - Ríos

Articulo Protegido por Licencia Creative Commons: BY-NC-ND / Protected by Creative Commons: BY-NC-ND.

Sendas es una revista de acceso abierto / Sendas is an Open Access Journal. 


\section{Discusión}

La variable actitudes ambientales estuvo conformado por 3 dimensiones, la misma que fue aplicada a los 304 estudiantes de la Facultad de Educación de la Universidad Nacional Mayor de San Marcos de Lima, de la tabla 2, se puede apreciar que, después de haber evaluado con el cuestionario de encuesta, se tiene que la gran mayoría de ellos que representa el $59,21 \%$ posee un nivel alto/bueno de conciencia ambiental, luego tenemos el $26,32 \%$ en el nivel regular, luego el $7,89 \%$ en el nivel deficiente, asimismo el $3,95 \%$ en el nivel muy bueno y finalmente solo el 2,63 en el nivel muy deficiente. Cabe mencionar que la gran mayoría de los estudiantes evaluados estuvo en el nivel alto el cual es favorable, tal como lo menciona Aguilar y Salas (2017).

De la tabla 3, se puede apreciar, que el nivel predominante de logro de la conciencia ambiental es el componente cognitivo luego el afectivo y finalmente el conductual. Lo cierto es que los estudiantes conocen muy bien el cuidado del medio ambiente, como menciona (Castro, 2012) son conscientes que el medio ambiente necesita, pero generalmente no lo practican, para el cual urge tomar medidas correctivas y preventivas.

Dichos, resultados son corroborados con los aportes de Ardaiz (2016), en su investigación Indicadores de desarrollo sostenible: la situación de Navarra, en donde concluye que en cada área los indicadores se clasifican por temas y subtemas, los cuáles merecen especial atención, principalmente en estudiantes en edad escolar.

Asimismo, la ubicación de un indicador en un área determinada no es excluyente para que esté relacionado con otras, de forma que podremos encontrar indicadores del ámbito social intrínsecamente ligados con los del ámbito económico.

Así mismo González (2016), en su investigación Medición del desarrollo sostenible a través de índices sintéticos: diseño y aplicación a la Unión Europea, concluyen que existe 4 componentes o dimensiones básicas de la sostenibilidad: institucional, medioambiental, económica y social, siendo la dimensión institucional, la que prevaleció y en el caso nuestro la parte cognitiva.

Asimismo, la conciencia ambiental es entender cómo influyen las acciones de cada

${ }^{\mathrm{a}}$ Dulio Oseda - Gago; ${ }^{\mathrm{b} J}$ Marco Antonio Oseda - Gago y ${ }^{\mathrm{c}}$ Ronald Henrry Toledo - Ríos

Articulo Protegido por Licencia Creative Commons: BY-NC-ND / Protected by Creative Commons: BY-NC-ND.

Sendas es una revista de acceso abierto / Sendas is an Open Access Journal. 
día en el medio ambiente y como esto afecta el futuro de nuestro espacio. Sin ser alarmista, conciencia ambiental, por ejemplo, es entender que si yo, ciudadano común, derrocho algún recurso natural, como puede ser el agua, mañana cuando quiera volver a utilizarlo ya no voy a poder. Muchas veces, al hablar de problemas ambientales se nos viene a la cabeza la destrucción de la capa de ozono, el calentamiento global, el alarmante aumento de la generación de residuos como mencionó (González, 2016).

Pero no vemos una relación directa entre nuestras acciones diarias y estos acontecimientos. Quizás, hablando sobre la eficiencia energética que tienen que lograr las industrias argentinas para no caer en una falta de suministro, estamos con todos los aparatos electrónicos que no estamos usando en standby, realizando un consumo sin mejorar en absoluto nuestra conformidad.

La conciencia ambiental se logra con educación a todos los niveles de la sociedad en todo momento en todo lugar. Hay que educar para poder concientizar. Desde el jardín de infantes hasta los abuelos/as. Todos tienen derecho a entender cuál es el problema ambiental y porque es importante la acción de cada uno de nosotros.
Existen en la actualidad muchos proyectos y planes donde distintos organismos e instituciones tanto públicas como privadas tratan de acercar información a los medios, a las universidades y a los ciudadanos. Mediante talleres prácticos se puede mostrar como es este delicado equilibrio entre seres humanos, naturaleza, entorno. Debemos entender en donde estamos sumergidos para comprender como es la relación y porqué debemos hacer todo lo que esté a nuestro alcance para protegerla precisó (Corraliza, T, et al., 2004).

Finalmente, en las universidades, colegios, centros de capacitación no solo es importante impartir conocimientos teóricos sobre la temática, sino también llevar adelante iniciativas y programas para que los alumnos y todos los que transitan por esos espacios vean la facilidad y la importancia de este tipo de acciones. Muchos proyectos ambientales requieren poco esfuerzo y nulos costos. Es una cuestión de decisión más que económica, el cual debe ser impulsado a corto y mediano plazo.

\section{Conclusiones}

1. Se ha determinado que el nivel predominante de la conciencia ambiental en los estudiantes de la

a Dulio Oseda - Gago; ${ }^{\mathrm{b} J}$ Marco Antonio Oseda - Gago y ${ }^{\mathrm{c}}$ Ronald Henrry Toledo - Ríos

Articulo Protegido por Licencia Creative Commons: BY-NC-ND / Protected by Creative Commons: BY-NC-ND.

Sendas es una revista de acceso abierto / Sendas is an Open Access Journal. 
Facultad de Educación de la Universidad Nacional Mayor de San Marcos de Lima fue el alto / bueno con el $59.21 \%$.

2. El componente más desarrollado de la conciencia ambiental fue el cognitivo con el 40,04\%, luego el componente afectivo con el $38,40 \%$ y finalmente el componente conductual con el $22,56 \%$, de donde se deduce que los estudiantes conocen muy bien el cuidado del medio ambiente, pero generalmente no lo practican.

\section{$\underline{\text { Referencias }}$}

Aguilar M. y Salas H. (2017) Informe sobre el medio mundial. Tratado de Kyoto. Bogotá: Mc Graw Hill.

Ander, E. (1984), Técnicas de Investigación Social. Buenos Aires: Paidós.

Ardaiz, I. (2016), Indicadores de desarrollo sostenible: la situación de Navarra. Instituto de Estadística de Navarra, Gobierno de Navarra.

Ary, D., et. al. (1993), Introducción a la investigación pedagógica. $2^{\circ}$ ed., México: Mc Graw - Hill.
Aye, L. y Widjaya, I. (2016) Educación y desarrollo. Bogotá: Mc Graw Hill.

Bermúdez, A. (2003) Actitudes hacia el desarrollo sostenible. México DF: Mc Graw Hill.

Betacourt, P., (2004) Educación ambiental. Bogotá: Mc Graw Hill.

Brack, E. (2012). Del área de Gestión Pedagógica, Especialista de Educación Ambiental implementa Normas específicas para la planificación, organización.

Castro, B. (2012), Indicadores de desarrollo sostenible urbano, la aplicación para Andalucía. Málaga: Universidad de Málaga en España.

Coll, C., et. al. (1995) El aprendizaje y la enseñanza de las actitudes. Los contenidos en la reforma enseñanza y aprendizaje de conceptos, procedimientos y actitudes. Madrid: Aula XXI Santillana.

CONAM (2017). Indicadores de desarrollo sostenible. Lima: MINSA.

Consejo Nacional del Ambiente (CONAM) (2008). Extraído el 31-08-08 del sitio web http://www.conam.gob.pe.

a Dulio Oseda - Gago; ${ }^{\mathrm{b} J}$ Marco Antonio Oseda - Gago y ${ }^{\mathrm{c}}$ Ronald Henrry Toledo - Ríos

Articulo Protegido por Licencia Creative Commons: BY-NC-ND / Protected by Creative Commons: BY-NC-ND.

Sendas es una revista de acceso abierto / Sendas is an Open Access Journal. 
2020.

URL: https://www.revistas.infoc.edu.pe/index.php/sendas

Email: revistasendas@infoc.edu.pe

Corraliza, T, et al. (2004) Educación para el futuro. Bogotá: Mc Graw Hill.

De Cruz, L. (2016) Reciclaje en la escuela básica Nacional. Mérida: Universidad Abierta de Mérida.

González, C. (2016), Medición del desarrollo sostenible a través de índices sintéticos: diseño y aplicación a la Unión Europea. Coruña: Departamento de Economía Aplicada de la Universidad de Coruña en España.

Hernández, R., Fernández, C. y Bautista, P. (2014) Metodología de la Investigación Científica. $6^{\circ}$ ed. México: Mc Graw Hill.

Kerlinger, F. y Lee, H. (2002) Investigación del comportamiento. México: Ed. Mc Graw. Hill. 3ra. ed.

Ley de Creación, Organización y funciones del Ministerio del Ambiente (2010): http://www.pucp.edu.pe/idea/docs/mi nam.pdf.

Ley $N^{\circ}$ 27314, Ley General de Residuos Sólidos.

López, M. (2017) Evaluación Integral para el Desarrollo Sostenido. Huancayo:
Vol. 1, N³, pp. 1-18 Septiembre 2020 ISSN $2708-6380$ https://doi.org/10.47192/rcs.v1i3.46

Escuela de Post Grado de la Universidad Nacional del Centro del Perú.

Morachimo, J. (2009) Indicadores de desarrollo sostenible. Bogotá: Mc Graw Hill.

Oseda, D. (2008) Metodología de la investigación. Huancayo: Pirámide.

Perú, Consejo Nacional del Ambiente (CONAM). (2002). Estrategia Nacional de Cambio Climático. Versión $\mathrm{N}^{\circ} 8$.

Perú, Consejo Nacional del Ambiente (CONAM). (2017). Manual para la Gestión de Residuos Sólidos en la Institución Educativa. Perú.

Rodríguez, R. (2007) Concepción didáctica para el tratamiento de la educación ambiental en secundaria básica. La Habana: Universidad de Cienfuegos de Cuba.

Rosales, K. (2017) Propuesta de un programa de actividades que facilite la construcción de una conciencia ecológica en los niños y niñas de 3 a 6 años. Buenos Aires: Paidós.

${ }^{\mathrm{a}}$ Dulio Oseda - Gago; ${ }^{\mathrm{b} J}$ Marco Antonio Oseda - Gago y ${ }^{\mathrm{c}}$ Ronald Henrry Toledo - Ríos

Articulo Protegido por Licencia Creative Commons: BY-NC-ND / Protected by Creative Commons: BY-NC-ND.

Sendas es una revista de acceso abierto / Sendas is an Open Access Journal. 
Sendas. 1(3), Julio - Septiembre, 2020.

URL: https://www.revistas.infoc.edu.pe/index.php/sendas

Email: revistasendas@infoc.edu.pe
Vol. 1, $\mathrm{N}^{\circ}$ 3, pp. 1-18 Septiembre 2020

ISSN $2708-6380$

https://doi.org/10.47192/rcs.v1i3.46

UNESCO (2017) Educación ambiental para

el desarrollo sostenible, Madrid:

Fondo editorial.

\section{(9) $\odot \Theta \Theta$}

Conciencia ambiental en estudiantes universitarios de una universidad pública de Lima (Dulio Oseda - Gago) Por Revista Sendas se encuentra bajo una Licencia Creative Commons-No Comercial-Sin Derivadas 3.0 Uported.

${ }^{\mathrm{a}}$ Dulio Oseda - Gago; ${ }^{\mathrm{b} J}$ Marco Antonio Oseda - Gago y ${ }^{\mathrm{c}}$ Ronald Henrry Toledo - Ríos

Articulo Protegido por Licencia Creative Commons: BY-NC-ND / Protected by Creative Commons: BY-NC-ND.

Sendas es una revista de acceso abierto / Sendas is an Open Access Journal. 